\title{
Simulation and Experiment of Extinction or Adaptation of Biological Species after Temperature Changes
}

\author{
D. Stauffer ${ }^{1}$ and H. Arndt ${ }^{2}$ \\ Cologne University, D-50923 Köln, Euroland \\ ${ }^{1}$ Institute for Theoretical Physics, e-mail: stauffer@thp.uni-koeln.de \\ 2 Zoological Institute, e-mail: hartmut.arndt@uni-koeln.de
}

\begin{abstract}
Can unicellular organisms survive a drastic temperature change, and adapt to it after many generations? In simulations of the Penna model of biological ageing, both extinction and adaptation were found for asexual and sexual reproduction as well as for parasex. These model investigations are the basis for the design of evolution experiments with heterotrophic flagellates.
\end{abstract}

Keywords: Penna model, evolution, population ecology, Monte Carlo

Global warming may be a relatively slow process, but the drastic temperature drop after a full-scale nuclear war ("nuclear winter") or its more speculative form shown in the Hollywood movie "The day after tomorrow" may cause the extinction of mankind. Though global warming may slowly change the mean temperature, it is expected that amplitudes of temperature fluctuations increase significantly. Example for adaptations to unusual temperatures are thermophilic bacteria near hot springs, or simple eukaryotes having adjusted to the higher temperatures in high-temperature sewage treatment plants. We plan biological experiments with heterotrophic flagellates (diploid and unicellular) to check how these protists survive a rapid or slow temperature change, by adaptation to the new environment over many generations. To guide these experiments which may take years we report here much simpler computer simulations of the same effect.

The Penna model [1, 2] is the most often used computer model for biological ageing. It represents the DNA genome by a computer word of, say, 32 bits such that bit = zero means a favourable ("wild type") allele while bit $=1$ represents a deleterious heritable mutation, starting to affect the health from that age on to which the bit position corresponds. Thus the whole lifespan is divided into 32 time units, which may correspond to several years for humans and less than an hour for bacteria and protists. At each time unit, each living adult beyond the minimum reproduction age of eight time units produces asexually one offspring with probability $b$. The offspring has the same genome as the parent except for one detrimental mutation at a random bit position; if the bit is already mutated it stays as it is. Three active bad mutations kill the individual. For sexual reproduction, each individual has two bit-strings, produces gametes by crossover, and one male and one female gamete are combined to allow the mother to give birth. For parasex of haploid bacteria [5], parts of the bit-strings of two individuals are exchanged, but no distinction between male and female is made. Further details including computer programs are given in [2], somewhat updated in [3]. We use the parameters of the programs listed there except that the birth rate $b$ is reduced to 0.3 . 
As in 4], a rapid change of the environment (here: temperature) is simulated by assuming that at one certain bit position, what was before a good allele (bit $=0$ ) now becomes bad, and what was bad (bit $=1$ ) now becomes good. (This might represent the production or suppression e.g. of heat shock proteins.) Technically for haploid genomes this is achieved by changing all the bits at this position. We simulated 20,000 time units, and changed the "temperature" at time step 10,000. The position of the bit which changes its meaning was typically 12 , slightly higher than the minimum reproduction age of 8 .

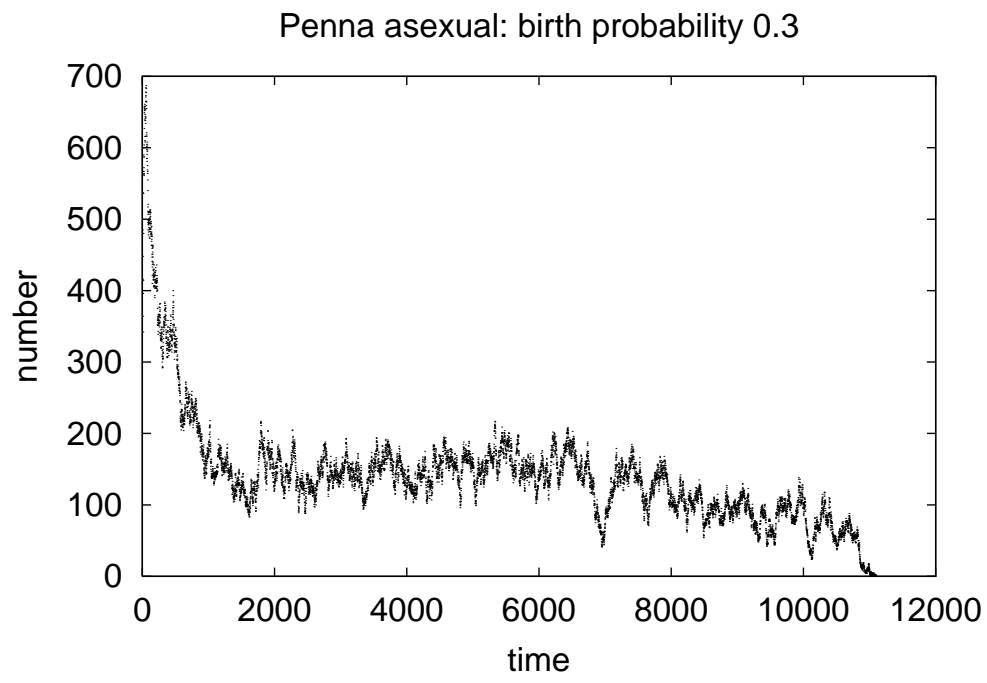

Figure 1: Extinction of an asexual population shortly after a change at time $=10,000$; birth rate 0.3 .

Fig. 1 shows that the temperature shock can lead to the extinction of the whole population 4. By starting with a larger population, this extinction can be avoided, Fig.2. Increasing the population further reduces the relative fluctuations, Fig.3. Also with parasex, Fig.2, or sexual reproduction, Fig.4, similar effects are observed. We see thus how the temperature change at first reduces the survival, and the population diminishes drastically. After some time, enough individuals who had the initially bad and later good mutation have produced enough offspring so that the total population recovers slowly.

Our biological experiments thus far are of the type of Fig.1; we still have to search for conditions where not the whole population dies out.

In conclusion these simulations suggest that high population densities $\left(\gg 10^{3}\right.$ individuals) and an experimental time consisting of hundreds of generations are necessary prerequisites to successfully study genetic adaptation to changed temperatures. The computational analysis indicated that the study of bacteria and nanoprotists (e.g. heterotrophic flagellates) should offer a suitable possibility for evolutionary experiments lasting only a few years.

Acknowledgements: We thank M. Lässig and D. Tautz for stimulating this cooperation. 




Figure 2: Survival of a larger population (x); otherwise parameters as in Fig.1. The upper $(+)$ symbols refer to the more favourable parasex.

\section{References}

[1] T.J.P. Penna, J. Stat. Phys. 78, 1629 (1995).

[2] S. Moss de Oliveira, P.M.C. de Oliveira and D. Stauffer, Evolution, Money, War and Computers (Teubner, Leipzig, 1999).

[3] D. Stauffer, The Complexity of Biological Ageing. p. 131 in: Thinking in Patterns, ed. by M.M. Novak, World Scientific, Singapore 2004.

[4] J.S. Sá Martins and S. Moss de Oliveira, Int. J. Mod. Phys. C 9, 421 (1998).

[5] S. Moss de Oliveira, D. Stauffer and P.M.C de Oliveira, Physica A 322, 521 (2003) 
Penna asexual, various poopulation sizes

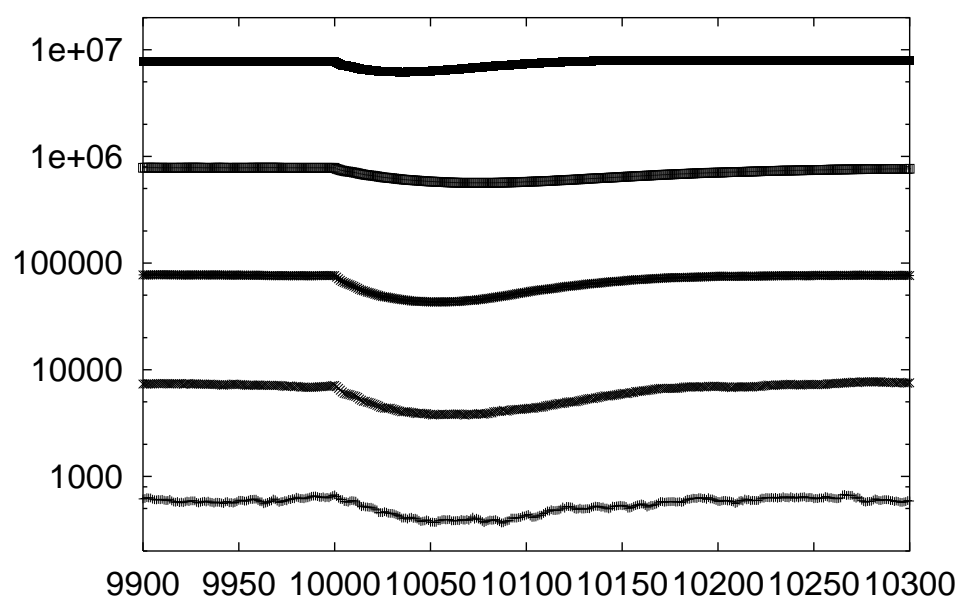

Figure 3: Dependence of fluctuations and systematic change on population size; otherwise parameters as in Figs. 1,2.

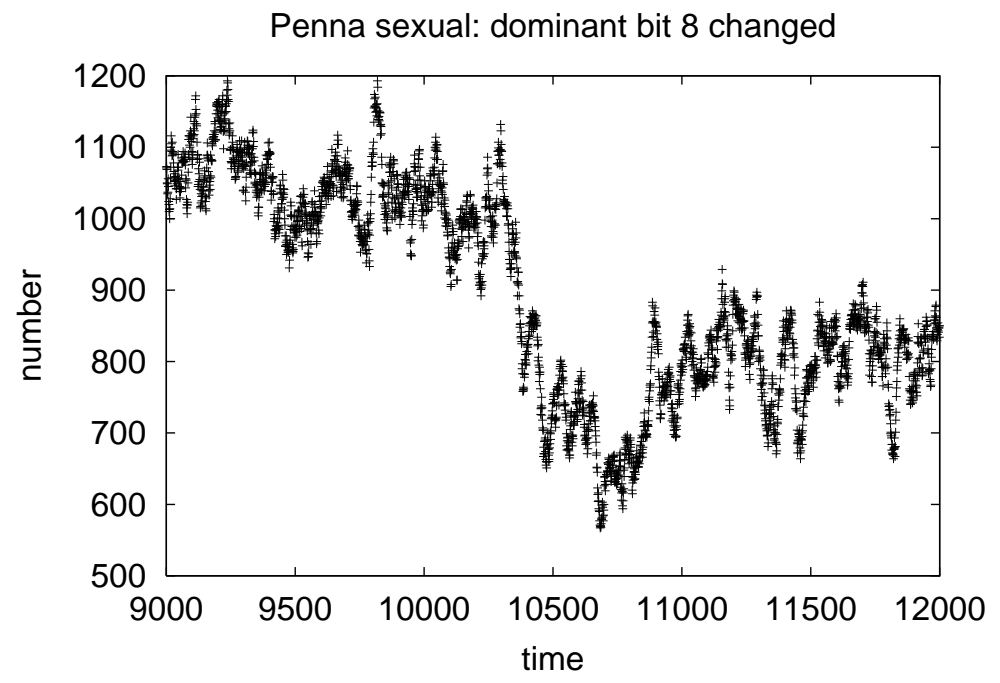

Figure 4: Effect on a sexual population. Birth rate 0.6 for females. Note expanded time interval shown. The changed bit was at position 8 and dominant. 